\title{
DFT Study on Structural, Electronic and Optical Properties of Ag-Doped SrTiO3 Perovskite for Optoelectronic Applications
}

\author{
Jalil Rehman ${ }^{1}$, M.Awais Rehman ${ }^{1}$, Muhammad Bilal Tahir ${ }^{1}$, Muhammad Usman ${ }^{1}$, and \\ Faisal Iqbal ${ }^{2}$ \\ ${ }^{1}$ KFUEIT \\ ${ }^{2} \mathrm{IUB}, \mathrm{BWP}$
}

May 27, 2021

\begin{abstract}
This study addresses the first-principles analysis using generalized gradient approximation (GGA), which is pillared on density functional theory (DFT), to find the effects of silver (Ag) doping on SrTiO3 structurally, electronically and optical properties. As Ag doping into SrTiO3, we see a small decrease in the volume of unit cell. Moreover, Ag-doping adds new states in SrTiO3 at Brillouin zone symmetry points, transferring host material's indirect band gap to a direct band gap. Ag doping in SrTiO3 results in the transfer density of states to smaller energies and increase in interaction among Ag atom and its surrounding atoms. Moreover, at the conduction band, the partial density of states (PDOS) of SrTiO3 changes generally. As a result, we conclude that Ag doping has an effect on the electronic band structure of SrTiO3. SrTiO3 doping with Ag has improved optical properties and its ability of converting to direct band gap results it in a perfect choice for optoelectronic applications.
\end{abstract}

\section{Introduction:}

Semiconductor materials are most important to use as photo catalyst to split water for production of refined hydrogen fuel $[1,2]$. Appropriate band positions are interesting parameters in splitting water by photo catalytic action. In other words, oxidation potential of water should be less positive than $\mathrm{V}_{\mathrm{BM}}$ valance band maxima and reduction potential of hydrogen must be less negative than $\mathrm{C}_{\mathrm{BM}}$ conduction band minima. $\mathrm{SrTiO}_{3}$ is significantly stable and has a large number of raw materials due to which it has sensational photo catalytic effect to split water and extract hydrogen with the help of solar energy. $\mathrm{SrTiO}_{3}$ having cubic structure is a perovskite material which has a wide range of applications as it has photo catalytic activity, being used to store energy, for gas sensing, in lithium-ion batteries as anode material, RAM (random access memory) and devices which deals with microwaves [3,4]. $\mathrm{SrTiO}_{3}$ can be prepared by several techniques like hydrothermal method [5], PLD, Sol-gel [6], coprecipitation method, electrospinning method [7], polymeric precursor technique etc. [8]. and a lot of work on its pure and metal doped samples have been done to study various physical properties. We performed all of the simulations CASTEP code, which is built on DFT and uses a plane wave pseudo potential approach with no approximation of the orbital form. In this article, structural analysis, optical properties and electronic properties determined. The band gap configuration plus lattice parameters of $\mathrm{Ag}$ doped $\mathrm{SrTiO}_{3}$ are also investigated. The existence of new states at G-(gamma) points was related to a minor variance in the lattice parameter of $\mathrm{Ag}$ doped $\mathrm{SrTiO}_{3}$. We've seen a red change in the optical properties of $\mathrm{SrTiO}_{3}$ through Ag-doping.

The following is the outline for this article: We include the computation information in section computational details. The section results and analysis contain the results and discussion. The article finally ends with the section of conclusion.

\section{Computational Details:}


$\mathrm{SrTiO}_{3}$ cubic structure belongs the $\mathrm{Pm} 3 \mathrm{~m}$ space group at room temperature. In this article electronic and structural properties of $\mathrm{SrTiO}_{3}$ plus its Ag doped sample were studied using technique of DFT. The CASTEP code was used for this, which made it simple to measure and compute the physical properties of a perovskite like $\mathrm{SrTiO}_{3}$. We research the impact of optical properties on dielectric constant in this software, as well as many other physical properties, but electrical, optical, structural, and electronic properties were our main concerns. First, we described and optimized the geometry of our perovskite, and then we used CASTEP to measure the physical properties of $\mathrm{SrTiO}_{3}[9,10]$. Ground state properties and pseudo-physical properties were discovered using DFT. K-points pieces of Brillouin zone testing for unmixed and mixed $\mathrm{SrTiO}_{3}$ were set at $2 \times 2 \times 1$ on the Monkhorst Pack grid [11-12].

\section{Results and Discussion}

\subsection{Structural Observation:}

To calculate the structural, optical properties and electronic properties of both pure $\mathrm{SrTiO}_{3}$ and $\mathrm{Ag}$ doped $\mathrm{SrTiO}_{3}$ compounds space group $\mathrm{Pm} 3 \mathrm{~m}$, as the position of atoms throughout the supercell of both pure $\mathrm{SrTiO}_{3}$ further $\mathrm{Ag}$ doped $\mathrm{SrTiO}_{3}$ as seen in figure seen in figure show shown in Fig 1 (a) \& (b) respectively Sr: $(0,0,0), \mathrm{Ti}:\left(\frac{1}{2}, \frac{1}{2}, \frac{1}{2}\right)$ and $\mathrm{O}:\left(\frac{1}{2}, 0, \frac{1}{2}\right)$.

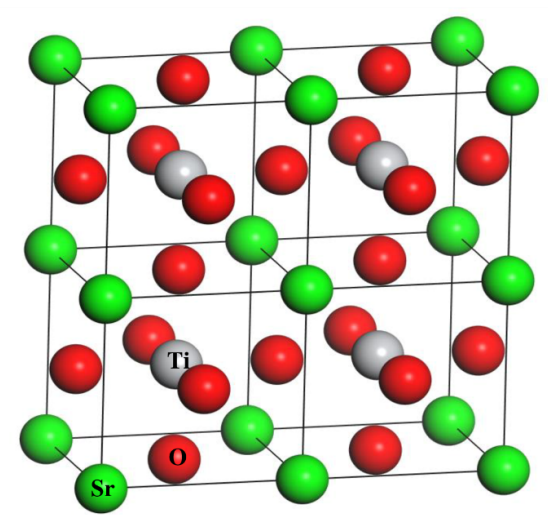

(a)

Fig 1: (a) Supercell of pure $\mathrm{SrTiO}_{3}$

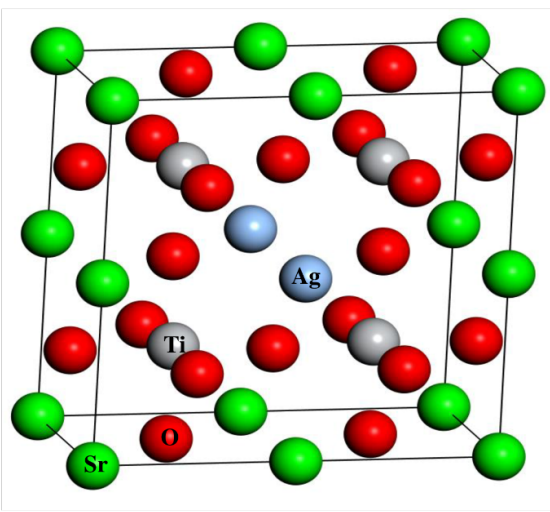

(b)

\subsection{Optimization of Geometry:}

The modified geometrical parameters for pure $\mathrm{SrTiO}_{3}, \mathrm{a}=\mathrm{b}=\mathrm{c}=3.944 \AA$, were achieved after the creativity of $2 \times 2 \times 1$ supercell and directly related with theoretical literature data [13]. Our estimated value is virtually identical to the previously published results, demonstrating the truthfulness of calculations of first-principles. The same method adopted to dope $\mathrm{SrTiO}_{3}$ with $\mathrm{Ag}$ atoms. The parameters of lattice were changed almost $0.0074 \AA$ for $\mathrm{SrTiO}_{3}$ and its $\mathrm{Ag}$ doped sample. In comparison to our calculated lattice parameters, As shown in Table 1 that the lattice parameters of $\mathrm{SrTiO}_{3}$ structure substantially overcalculated ( $\mathrm{a}, \mathrm{b}$ and $\mathrm{c}=3.937 \AA$ ) due to $\mathrm{Ag}$ doping. $\mathrm{Ag}$ doping in $\mathrm{SrTiO}_{3}$ also causes a decrease in the volume of the supercell. This is $\mathrm{Ag}$ has ionic radius of $0.128 \mathrm{~nm}$ which is larger than that of $\mathrm{Sr}$ which is $0.113 \mathrm{~nm}$.

\subsection{Electronic properties of pure $\mathrm{SrTiO}_{3}$ and $\mathrm{Ag}$ doped $\mathrm{SrTiO}_{3}$ :}



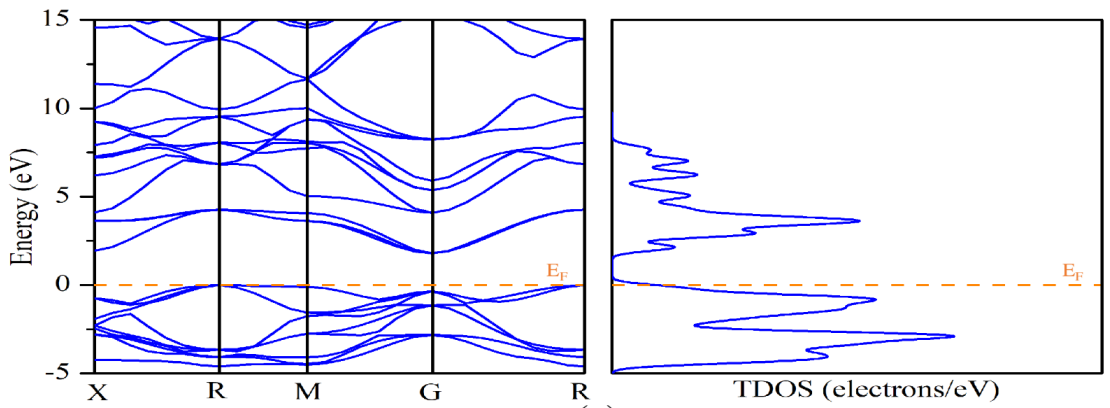

(a)
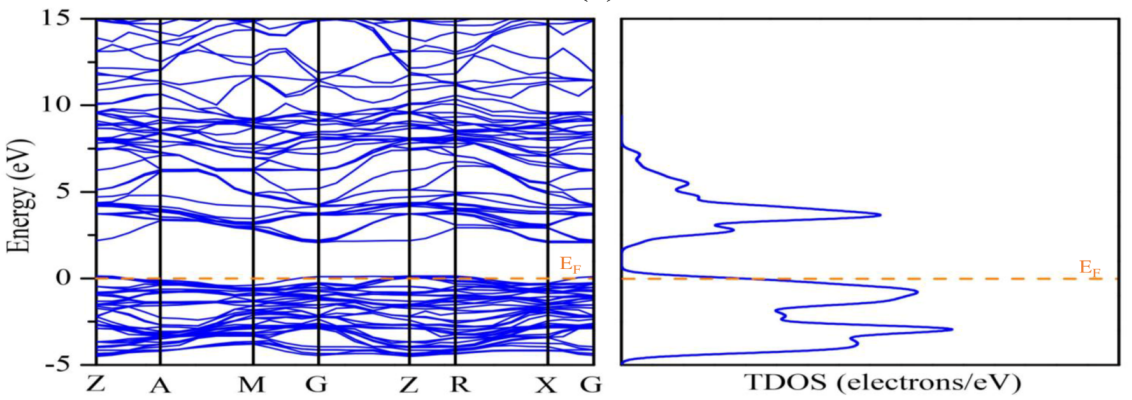

(b)

Fig 2: (a) Band structure and $\mathrm{TDOS}_{\text {of }}$ pure $\mathrm{SrTiO}_{3}$

(b) Band structure and TDOS of Ag-doped $\mathrm{SrTiO}_{3}$

\subsubsection{Band Structure}

We measured the band structures of pure $\mathrm{SrTiO}_{3}$ as well as effect for Ag atom doping upon the electronic properties of $\mathrm{SrTiO}_{3}$. The band structure of both pure and doped $\mathrm{SrTiO}_{3}$ are shown in Fig 2 (a) \& (b), respectively. We can see that maximum of valance band (VB) present at point $\mathrm{R}$ and the minima of conduction band $(\mathrm{CB})$ present at point $\mathrm{G}$, which confirms the indirect band gap of pure $\mathrm{SrTiO}_{3}$. Taking such two approximation points into consideration, the calculated bandgap of pure $\mathrm{SrTiO}_{3}$ is $1.91 \mathrm{eV}$ and Ag-doped $\mathrm{SrTiO}_{3}$ is $2.18 \mathrm{eV}$. This indirect band gap findings are nearly matched with previous study. As we can see that in the case of pure $\mathrm{SrTiO}_{3}$, the value of direct band gap is lesser than the value which is obtained by experiment which is $3.2 \mathrm{eV}$ [22]. From Fig 2(b), both minima of conduction band and the maxima of the valence band present at same point "G" throughout the observed results of Ag-doped $\mathrm{SrTiO}_{3}$ and after doping the indirect band gap was converted to direct band gap. The graph reveals that the VB of $\mathrm{Ag}-\mathrm{SrTiO}_{3}$, especially at point $\mathrm{G}$, is transferred up than that of pure $\mathrm{SrTiO}_{3}[15,16]$.

\begin{tabular}{|c|c|c|c|c|c|}
\hline & $\begin{array}{l}\text { Parameters of } \\
\text { lattice }(\AA)\end{array}$ & $\begin{array}{l}\text { Parameters of } \\
\text { lattice }(\AA)\end{array}$ & $\begin{array}{l}\text { Parameters of } \\
\text { lattice }(\AA)\end{array}$ & Volume $\left(\mathrm{A}^{3}\right)$ & $\begin{array}{l}\text { Band Gap } \\
(\mathrm{eV})\end{array}$ \\
\hline & $\mathrm{a}$ & $\mathrm{b}$ & $\mathrm{c}$ & & \\
\hline $\begin{array}{l}\text { Previously } \\
\text { Reported } \\
\left(\text { Pure } \mathrm{SrTiO}_{3}\right) \\
{[13]}\end{array}$ & 3.875 & 3.875 & 3.875 & 58.186 & 2.24 \\
\hline $\begin{array}{l}\text { Previously } \\
\text { Reported } \\
\text { (Ag-doped) } \\
{[14]}\end{array}$ & 3.892 & 3.892 & 3.892 & 58.955 & 2.40 \\
\hline $\begin{array}{l}\text { Present Study } \\
\left(\text { Pure } \mathrm{SrTiO}_{3}\right)\end{array}$ & 3.944 & 3.944 & 3.944 & 61.368 & 1.91 \\
\hline
\end{tabular}




\begin{tabular}{llllll}
\hline & $\begin{array}{l}\text { Parameters of } \\
\text { lattice }(\AA)\end{array}$ & $\begin{array}{l}\text { Parameters of } \\
\text { lattice }(\AA)\end{array}$ & $\begin{array}{l}\text { Parameters of } \\
\text { lattice }(\AA)\end{array}$ & Volume $\left(\mathrm{A}^{3}\right)$ & $\begin{array}{l}\text { Band Gap } \\
(\mathrm{eV})\end{array}$ \\
\hline $\begin{array}{l}\text { Present Study } \\
\text { (Ag-doped) }\end{array}$ & 3.937 & 3.937 & 3.937 & 61.023 & 2.18 \\
\hline
\end{tabular}

Table 1: Lattice parameters and band gap of pure $\mathrm{SrTiO}_{3}$ and Ag-doped $\mathrm{SrTiO}_{3}$ :

3.3.2 Partial Density of State (PDOS) and Total Density of State (TDOS):
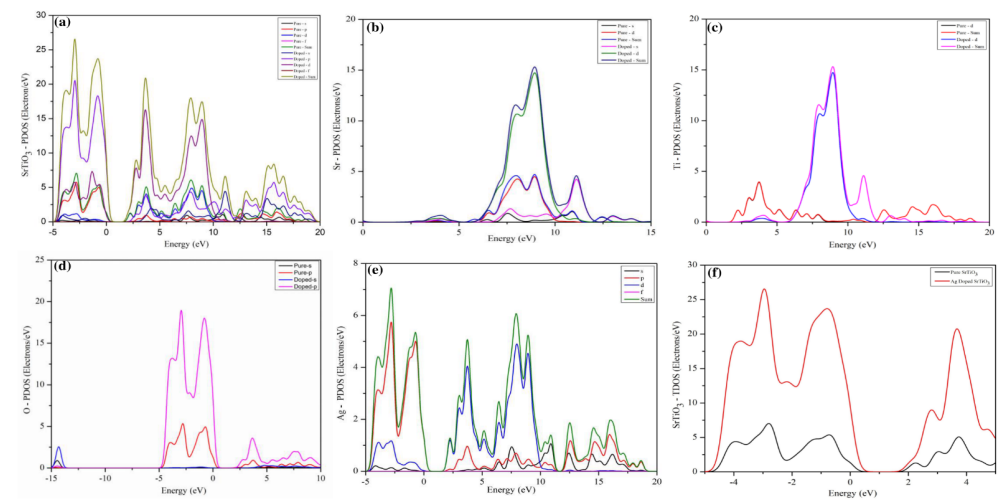

Fig 3: (a) PDOS for pure and Ag doped SrTiO (c) PDOS of Ti before and after Ag doping

(b) PDOS of Sr before and after Ag doping (e) PDOS of A

(d) PDOS of $O$ before and after $A g$ doping (f) TDOS for pure and $\mathrm{Ag}$ doped $\mathrm{SrTiO}_{3}$

We can see that the upper position of the valence band has major influence after doping. Furthermore, the O-p states and s-DOS on either side the Fermi stage tend to be sharpen by the $\mathrm{O}$ atom after Ag doping in present lattice. Fig 3 (a) shows the PDOS of pure $\mathrm{SrTiO}_{3}$ and $\mathrm{Ag}$-doped $\mathrm{SrTiO}_{3}$ we observed that the value of $\mathrm{Ag}$-doped $\mathrm{SrTiO}_{3}$ is maximum as compared to pure $\mathrm{SrTiO}_{3}$. The elemental PDOS was plotted to compare the pure and doped systems Fig 3 (b \& e) that exhibit major differences in valance and conduction band states before doping and after doping. Fig 3 (c) shows the PDOS of pure and Ag-doped $\mathrm{Ti}$, we observed that value of $\mathrm{Ag}$-doped $\mathrm{Ti}$ is greater as compare to pure Ti. Fig 3 (f) shows the TDOS and for pure and $\mathrm{Ag}$-doped $\mathrm{SrTiO}_{3}$, we see that the top at the valence band is in the limit of 0 to 5 electron Volt generates deep effect, according to the TDOS, due to recently emerging Ag-d states. By appearance the Ag- d states at the upper point of the valence band at point $\mathrm{G}$ could explained an upper change in the valance band. As a result, we claimed that $\mathrm{Ag}$ doping in $\mathrm{SrTiO}_{3}$ has two significant consequences. The first shows the reduction in band gap reasoned of inclusion of $\mathrm{Ag}$ in $\mathrm{SrTiO}_{3}$. Second, we see alter in structure of the band gap after doping from indirect to direct. As a result, $\mathrm{Ag}$ doped $\mathrm{SrTiO}_{3}$ would be much effective for optical devices $[17,18]$.

\subsection{Optical Properties:}



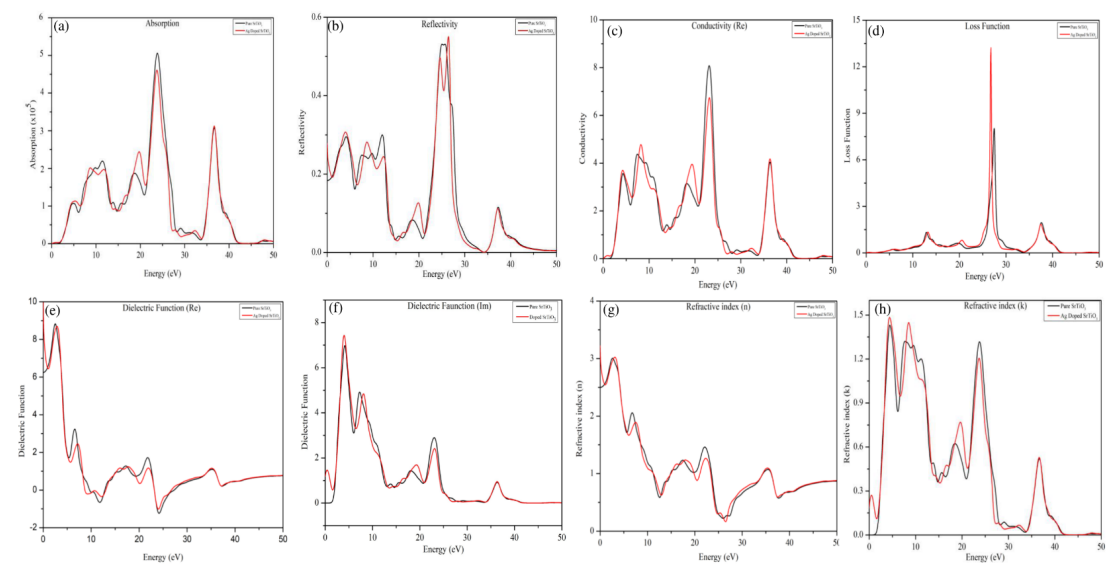

Fig 4: A comparison of the optical properties of pure (Black) and Ag doped (Red) $\mathrm{SrTiO}_{3}$
$\begin{array}{lllll}\text { (a) Absorption } & \text { (b) Reflectivity } & \text { (c) Conductivity (real part) } & \text { (d) Loss Function } & \text { (e) Dielectric Function } \\ \text { (real part) } & \text { (f) Dielectric Function (imaginary part) } & \text { (g) Refractive index (n) } & \text { (h) Refractive index (k) }\end{array}$

Since $\mathrm{SrTiO}_{3}$ is energetic compound of optical electronic devices, we examine result $\mathrm{Ag}$ doping with $\mathrm{SrTiO}_{3}$ lattice. Properties of optical such as reflectivity, absorption, refractive index (n), refractive index $(\mathrm{k})$, loss function, real part and imaginary part of dielectric function and real part of conductivity describe how light interact with matter. Firstly, we calculated these optical properties of pure $\mathrm{SrTiO}_{3}$ compound and after this we compare the optical properties with Ag doped SrTiO3 compound shown in Fig 4. We analysis that these optical properties are frequency dependent and interconnected.

$\mathrm{Ag}$ doped $\mathrm{SrTiO}_{3}$, all points where the absorption is lowest, the reflection Fig 4(b) is found to be maximum. Here we analyze that the presence of doping slightly changes the absorption spectra.

As shown in Fig 4(e \& f) the dielectric function is divided into two parts: First is real part and second is imaginary part. The real part of dielectric function (DF) represents polarization, while the imaginary part describes energy dissipation within the framework. In the case of a pure $\mathrm{SrTiO}_{3}$, the imaginary component of $\mathrm{DF}$ is 0 at 0 electron Volt. It illustrates that energy dissipation (absorption) is zero inside the $\mathrm{SrTiO}_{3}$. If we compare this value with the Ag-doped $\mathrm{SrTiO}_{3}$ we observe that energy dissipation is present at 0 electron Volt. The imaginary part of DF shows four highest peaks which observed at 4.12, 7.28, 23.02 and 36.33 electron Volt of pure $\mathrm{SrTiO}_{3}$, which relates the 4 absorption peaks depicted in Fig 4(a). Ag-doped $\mathrm{SrTiO}_{3}$ shows notable peak at $8.69 \mathrm{eV}$ and absorption peak at 23.80 electron Volt is slightly decreases for doped $\mathrm{SrTiO}_{3}$. It can be observed $\mathrm{Ag}$-doped $\mathrm{SrTiO}_{3}$ system indicates do not a change in absorption peaks from high energy to low energy, but also a transfer in absorption peaks away from high energy. The energy area in which electrons do not generally bound to their lattice positions and conduct plasma oscillations upon light exposure defined as the maximum loss function. In comparison to the pure $\mathrm{SrTiO}_{3}$, we analyze that the Ag-doped $\mathrm{SrTiO}_{3}$ a high peak of plasma oscillation has shifted to smaller energies. Fig 4(d) shows that the energy loss function of doped $\mathrm{SrTiO}_{3}$ reaches its maximum value as compare to pure system of the dielectric function. The both parts of refractive index ( $\mathrm{n} \& \mathrm{k}$ ), which based on energy (frequency), make complex refractive index shown in Fig $4(\mathrm{~g} \& \mathrm{~h})$. The doped system's refractive index (n) is estimated to be $3.23 \mathrm{eV}$, which is significantly higher than the pure system's $(2.50 \mathrm{eV})$. After Ag addition, the refractive index (n) of semiconducting $\mathrm{SrTiO}_{3}$ shifts to higher values, confirming the transition of semiconducting $\mathrm{SrTiO}_{3}$ to metallic material. The lowest absorption energy is correlated with highest refractive index value at zero photon energy. The refractive index decreases as absorption increases, as seen in Fig 4(g). The annihilation of energy in the system defined be with extinction coefficient, which correlated by the absorption spectrum. At photon energy is $1.3 \mathrm{eV}$, the extinction coefficient of pure $\mathrm{SrTiO}_{3}$ is zero, which is the same as its indirect band gap and until $1.3 \mathrm{eV}$, there is no extinction of energy inside the substance. So, that it has zero absorption. The refractive index (n) rises in parallel by absorption. The refractive index (k) of Ag-doped $\mathrm{SrTiO}_{3}$ has transferred to a lower energy, with sharp peaks occurring at about $4.40 \mathrm{eV}, 8.50 \mathrm{eV}, 19.65 \mathrm{eV}, 23.68 \mathrm{eV}$ and $36.41 \mathrm{eV}$ [19-21]. 


\section{Conclusion:}

In the recent work we applied the first-principles calculations on the basis of DFT to explore the outcomes of $\mathrm{Ag}$ on structural analysis, optical and electronic properties of $\mathrm{SrTiO}_{3}$ compound. Our calculated structures match those reported in the literatures and are discussed in the context of Ag-doping. Before doping and after doping of $\mathrm{Ag}$, we measured the band structure of $\mathrm{SrTiO}_{3}$. Furthermore, using the concepts of TDOS and PDOS, the "band structure" both events are thoroughly investigated. After Ag doping, we observe that the Fermi level shifts near to conduction band, similar to activity of n-type degenerate semiconductors. With comparison of pure $\mathrm{SrTiO}_{3}$, the introduction of new Ag DOS at G points leads to the decrease in optical band gap. Moreover, at the conduction band, the partial density of states (PDOS) of $\mathrm{SrTiO}_{3}$ changes generally. As a result, we conclude that $\mathrm{Ag}$ doping has an effect on the electronic band structure of $\mathrm{SrTiO}_{3} \cdot \mathrm{SrTiO}_{3}$ doping with Ag has improved optical properties and its ability of converting to direct band gap results it in a perfect choice for optoelectronic applications.

\section{References:}

1. Tong, H., Ouyang, S., Bi, Y., Umezawa, N., Oshikiri, M., \& Ye, J. (2012). Nano-photocatalytic materials: possibilities and challenges. Advanced materials, 24(2), 229-251.

2. Chen, X., Shen, S., Guo, L., \& Mao, S. S. (2010). Semiconductor-based photocatalytic hydrogen generation. Chemical reviews, 110(11), 6503-6570.

3. Reunchan, P., Ouyang, S., Umezawa, N., Xu, H., Zhang, Y., \& Ye, J. (2013). Theoretical design of highly active SrTiO 3-based photocatalysts by a codoping scheme towards solar energy utilization for hydrogen production. Journal of Materials Chemistry A, 1(13), 4221-4227.

4. Van Benthem, K., Elsässer, C., \& French, R. H. (2001). Bulk electronic structure of SrTiO 3: Experiment and theory. Journal of applied physics, 90(12), 6156-6164.

5. Niishiro, R., Tanaka, S., \& Kudo, A. (2014). Hydrothermal-synthesized SrTiO3 photocatalyst codoped with rhodium and antimony with visible-light response for sacrificial $\mathrm{H} 2$ and $\mathrm{O} 2$ evolution and application to overall water splitting. Applied Catalysis B: Environmental, 150, 187-196.

6. Zhang, Y., Hu, J., Cao, E., Sun, L., \& Qin, H. (2012). Vacancy induced magnetism in SrTiO3. Journal of magnetism and magnetic materials, 324(10), 1770-1775.

7. Zhang, W., Li, H. P., \& Pan, W. (2012). Ferromagnetism in electrospun Co-doped SrTiO 3 nanofibers. Journal of Materials Science, 47(23), 8216-8222.

8. Chang, C. H., \& Shen, Y. H. (2006). Synthesis and characterization of chromium doped SrTiO3 photocatalyst. Materials Letters, 60(1), 129-132.

9. Clark, S. J., Segall, M. D., Pickard, C. J., Hasnip, P. J., Probert, M. I., Refson, K., \& Payne, M. C. (2005). First principles methods using CASTEP. Zeitschrift für Kristallographie-Crystalline Materials, 220(5-6), 567-570.

10. Mostaghni, F., \& Abed, Y. (2015). First-Principles Study on Anatase Co/TiO2: Effect of Co Concentration. Physical Chemistry, 5(2), 34-38.

11. Milman, V., Winkler, B., White, J. A., Pickard, C. J., Payne, M. C., Akhmatskaya, E. V., \& Nobes, R. H. (2000). Electronic structure, properties, and phase stability of inorganic crystals: A pseudopotential plane-wave study. International Journal of Quantum Chemistry, 77(5), 895-910.

12. Aboub, Z., Daoudi, B., \& Boukraa, A. (2020). Theoretical study of Ni doping SrTiO3 using a density functional theory [J]. AIMS Materials Science, 7(6), 902-910.

13. Qiu, B., Yan, X. G., Huang, W. Q., Huang, G. F., Jiao, C., Zhan, S. Q., .. \& \&eng, P. (2014). The Electronic and Optical Properties of X-Doped $\mathrm{SrTiO} 3(\mathrm{x}=\mathrm{Rh}, \mathrm{Pd}, \mathrm{Ag})$ : a First-Principles Calculations. International Journal of Modern Physics B , 28 (09), 1450031.

14. Qiu, B., Yan, X. G., Huang, W. Q., Huang, G. F., Jiao, C., Zhan, S. Q., .. \& \& Peng, P. (2014). The Electronic and Optical Properties of X-Doped $\mathrm{SrTiO} 3(\mathrm{x}=\mathrm{Rh}, \mathrm{Pd}, \mathrm{Ag})$ : a First-Principles Calculations. International Journal of Modern Physics B , 28 (09), 1450031.

15. Birch, F. (1947). Finite elastic strain of cubic crystals. Physical review, 71(11), 809.

16. Murnaghan, F. D. (1944). The compressibility of media under extreme pressures. Proceedings of the national academy of sciences of the United States of America, 30(9), 244. 
17. Irie, H., Maruyama, Y., \& Hashimoto, K. (2007). Ag+-and Pb2+-doped SrTiO3 photocatalysts. A correlation between band structure and photocatalytic activity. The Journal of Physical Chemistry C, 111(4), 1847-1852.

18. Rizwan, M., Ali, A., Usman, Z., Khalid, N. R., Jin, H. B., \& Cao, C. B. (2019). Structural, electronic and optical properties of copper-doped SrTiO3 perovskite: A DFT study. Physica B: Condensed Matter, 552, 52-57.

19. Park, H. S., Kim, D. H., Kim, S. J., \& Lee, K. S. (2006). The photocatalytic activity of 2.5 wt\% Cu-doped $\mathrm{TiO} 2$ nano powders synthesized by mechanical alloying. Journal of Alloys and Compounds, 415(1-2), 51-55.

20. Janotti, A., Jalan, B., Stemmer, S., \& Van de Walle, C. G. (2012). Effects of doping on the lattice parameter of SrTiO3. Applied Physics Letters, 100(26), 262104.

21. Philipp, H. R., \& Ehrenreich, H. (1962). Observation of d bands in 3-5 semiconductors. Physical Review Letters, 8(3), 92.

22. Zhao, L., Fang, L., Dong, W., Zheng, F., Shen, M., \& Wu, T. (2013). Effect of charge compensation on the photoelectrochemical properties of Ho-doped SrTiO3 films. Applied Physics Letters , 102 (12), 121905.

\section{Hosted file}

Title.doc available at https://authorea.com/users/416085/articles/523775-dft-studyon-structural-electronic-and-optical-properties-of-ag-doped-srtio3-perovskite-foroptoelectronic-applications

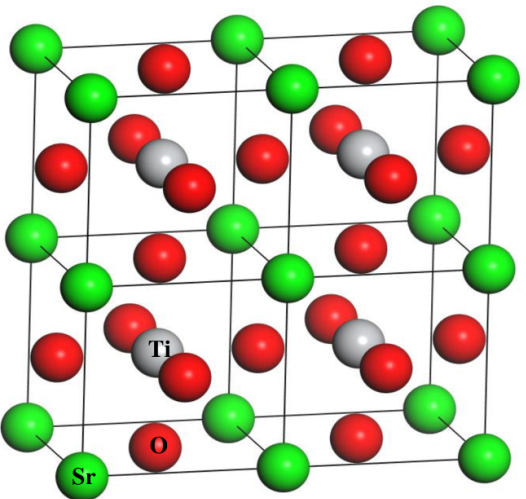

(a)

Fig 1: (a) Supercell of pure $\mathrm{SrTiO}_{3}$

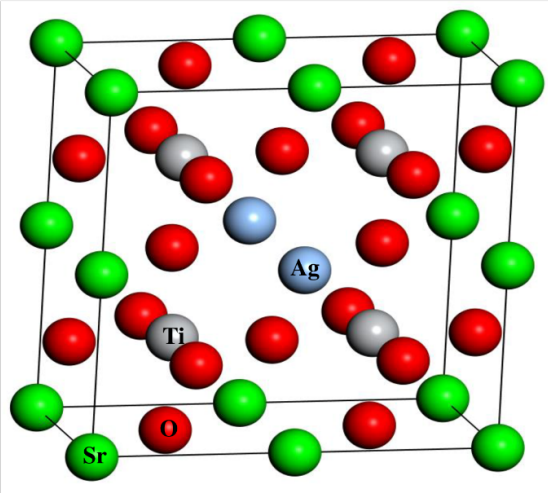

(b)

(b) Supercell of Ag-doped $\mathrm{SrTiO}_{3}$ 

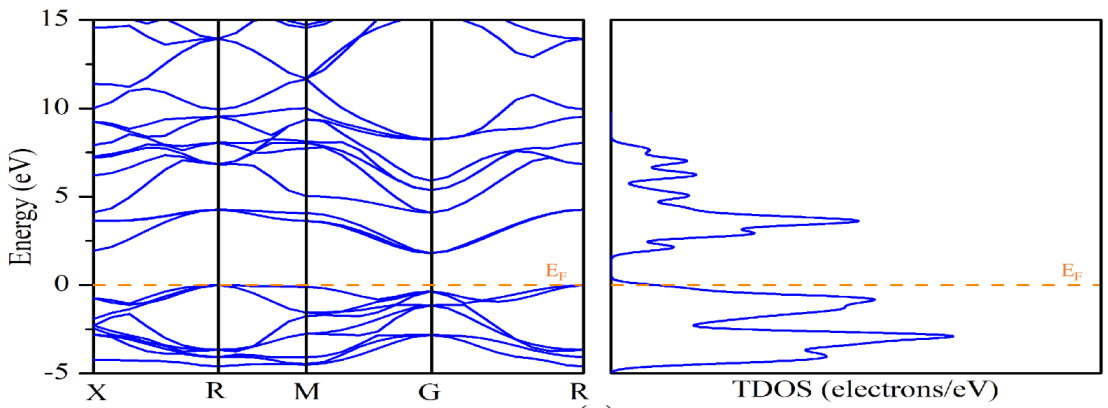

(a)
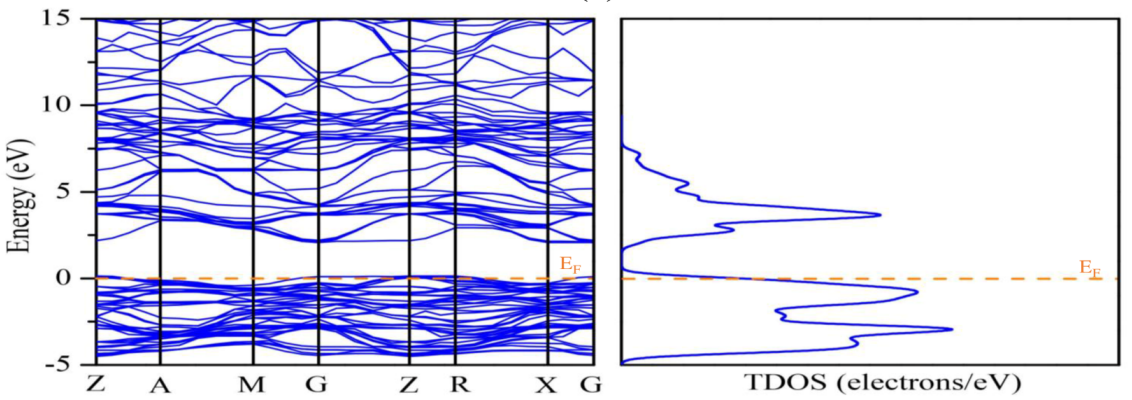

(b)

Fig 2: (a) Band structure and TDOS of pure $\mathrm{SrTiO}_{3}$

(b) Band structure and TDOS of Ag-doped $\mathrm{SrTiO}_{3}$
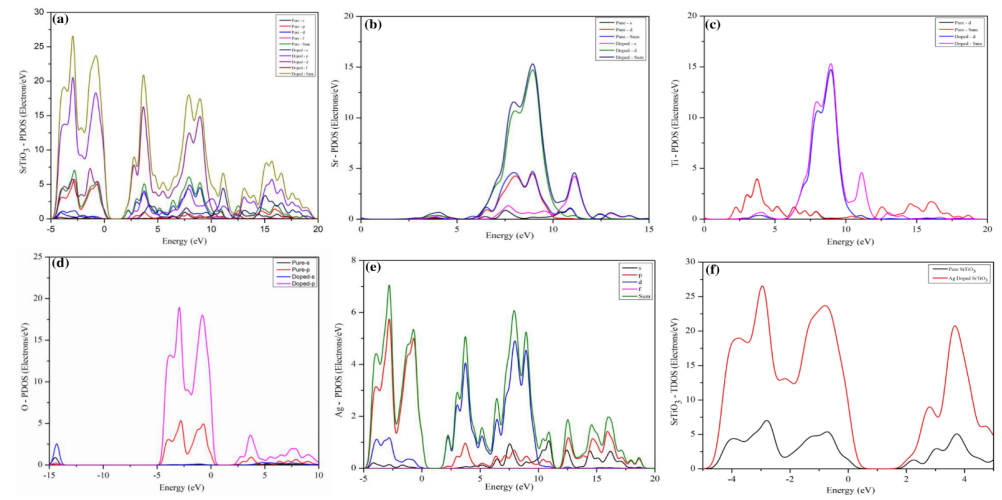

Fig 3: (a) PDOS for pure and $\mathrm{Ag}$ doped $\mathrm{SrTiO}_{3}$ (c) PDOS of Ti before and after $\mathrm{Ag}$ doping

(b) PDOS of $\mathrm{Sr}$ before and after $\mathrm{Ag}$ doping (e) PDOS of Ag

(d) PDOS of $\mathrm{O}$ before and after $\mathrm{Ag}$ doping (f) TDOS for pure and $\mathrm{Ag}$ doped $\mathrm{SrTiO}_{3}$ 

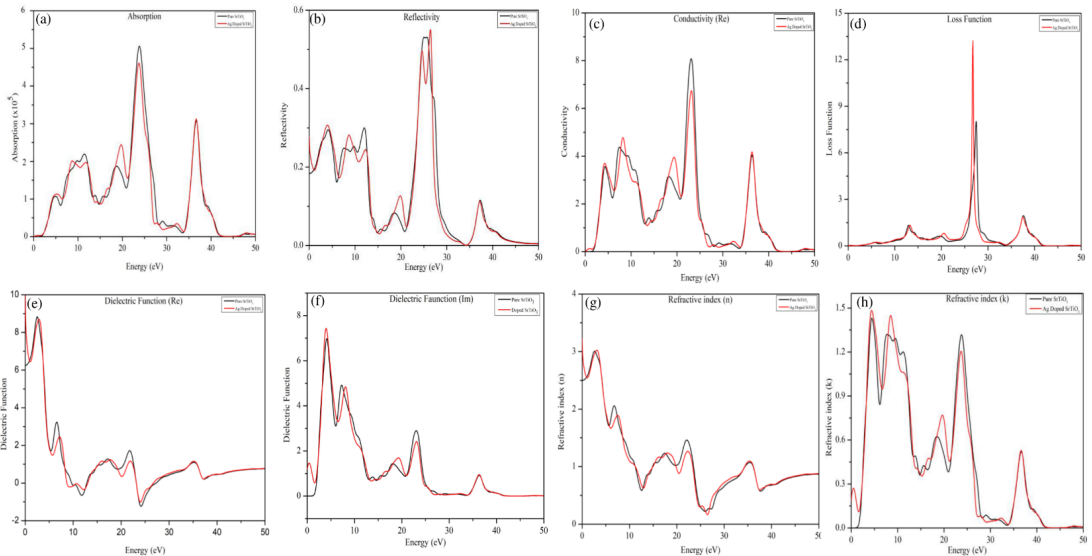

Fig 4: A comparison of the optical properties of pure (Black) and Ag doped (Red) $\mathrm{SrTiO}_{3}$

$\begin{array}{llll}\text { (a) Absorption (b) Reflectivity (c) Conductivity (real part) (d) Loss Function (e) Dielectric Function } & \text { (c) }\end{array}$ (real part) (f) Dielectric Function (imaginary part) (g) Refractive index (n) (h) Refractive index (k) 